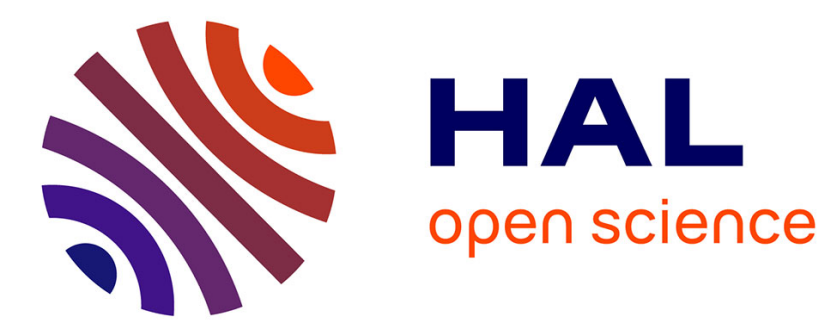

\title{
Le théâtre des passions politiques
}

Raymond Jamous

\section{To cite this version:}

Raymond Jamous. Le théâtre des passions politiques. Terrain : revue d'ethnologie de l'Europe , 2004, 43, pp.141-156. 10.4000/terrain.1877 . halshs-01938040

\section{HAL Id: halshs-01938040 https://shs.hal.science/halshs-01938040}

Submitted on 28 Nov 2018

HAL is a multi-disciplinary open access archive for the deposit and dissemination of scientific research documents, whether they are published or not. The documents may come from teaching and research institutions in France or abroad, or from public or private research centers.
L'archive ouverte pluridisciplinaire HAL, est destinée au dépôt et à la diffusion de documents scientifiques de niveau recherche, publiés ou non, émanant des établissements d'enseignement et de recherche français ou étrangers, des laboratoires publics ou privés. 


\title{
Le théâtre des passions politiques
}

\author{
Raymond Jamous
}

p. 141-156

\section{Résumés}

\section{Français \\ English}

Au Liban, les passions politiques se manifestent par une mise en scène des liens de domination, de clientèle, de compétition, l'exercice du pouvoir n'étant qu'un aspect exacerbé des rapports entre violence verbale et violence physique qui sont inscrites dans toutes les strates de la société. Différents personnages sont présentés à travers leurs performances : le jeune tueur, le beau parleur, le « leader des jeunes », le chef de famille et le seigneur qui est tenté de défier le ciel. Les trois premiers cherchent à s’imposer par leur action et/ou leur maîtrise de la parole en n'ayant qu'une influence limitée sur leurs agnats alors que les deux derniers agissent violemment en s'appuyant sur les relations de dépendance et en manifestant leur volonté de pouvoir. Par sa parole, l'opinion tempère ou exacerbe les tensions politiques, mais elle n'offre aucun moyen de légitimer ou de contrôler les échanges de violence et les affrontements qui ont dérapé et provoqué les massacres de la guerre civile.

The theater of political passions In Lebanon, political feelings are expressed through relations based on domination, patronage or competition. Exercising power is but one (intense) aspect of the relation between the violence in words and in deeds running through all social strata. Various figures are presented: the young killer, the smooth talker, the "youth leader", the head of family and the warlord who is tempted to defy heaven. The first three try to prevail through their actions or their control over language, but have limited influence over agnates. The last two act violently, manifest their will to power and use relations based on dependence. The words used in opinions moderate or exacerbate political tensions, but they offer no means for legitimating or controlling the acts of violence and clashes that, out of hand, resulted in massacres during the civil war.

Haut de page

Entrées d'index

Thèmes :émotions, politique (anthropologie)

Lieux d'étude :Liban

Mots-clés :honneur, Liban, narration comme action politique, pouvoir, violence verbale

Keywords :honor, Lebanon, narration as political action, power, verbal violence Haut de page

Plan

\section{Les jeunes dans leurs excès}


Destin de tueur $\bullet$
Le beau parleur $\bullet$
Le «leader des jeunes » ou sheikh ash-shebab
La recherche de prestige et de pouvoir
Le qabaday, I'« homme fort »
La volonté de pouvoir chez les seigneurs $\bullet$
La politique comme mise en scène de la violence

Avouez, tout de même, que la parole a chez nous un pouvoir de jouissance et d'extermination sans nom ! Dominique Eddé

La mise en scène des passions politiques est très répandue dans le pourtour méditerranéen. Ce théâtre se produit devant un public, qui est complice ou critique de la performance des acteurs, et dont les interprétations à travers les récits peuvent infléchir le sens de l'action et conduire à la violence. Il n'a pas d'auteur, de metteur en scène, ou de scénario préétabli. Par ailleurs, les acteurs ne peuvent pas enlever leur costume de scène et cesser de jouer, comme au vrai théâtre ou dans le rituel, car si la vie publique est un spectacle, les enjeux sont réels: la vie, la mort, I'honneur, le prestige, le pouvoir sur les hommes. Il existe des comportements réglés qui vont tellement de soi qu'ils n'ont pas besoin d'être formulés et qu'il faut chercher dans les différents récits et spectacles de la violence physique et du jeu de la parole. C'est à travers l'analyse de ces processus que l'on peut étudier une logique d'ensemble, une structure des passions politiques.

1 Cf. I'article de M. Johnson (1977) sur la position et le rôle des zu'ama (singulier : za'im) urbain (...)

Dans cette perspective, je m'inspirerai de l'ouvrage de Michael Gilsenan sur le Liban (Gilsenan 1996). Celui-ci décrit la situation dans des villages de la région du Akkar (Nord-Liban) en 1971, à la veille de la guerre civile. Dans cette région, une stratification sociale semble bien établie : des seigneurs (les beys), hommes de pouvoir et grands propriétaires terriens ; leurs clients (les aghas) qui leur servent de gardes et de gestionnaires de leur domaine ; et enfin les paysans (les fellahs) qui travaillent la terre des premiers sous la surveillance des seconds. Cette répartition tripartite de la population (les chefs politiques, les clients et les autres, ici les paysans) se retrouve ailleurs au Liban, même si elle prend des formes différentes selon les communautés et le milieu rural ou urbain 1. L'intérêt de cet ouvrage va bien au-delà de la description distanciée des règles qui régissent en apparence cette stratification sociale et des transformations que la région et le pays ont 
connues durant le xxe siècle. Il permet de saisir comment les trois strates sociales jouent différentes partitions et improvisations sur la scène politique et comment les rapports entre les strates entremêlent des liens de domination, de clientèle, de conflit, de compétition, de rivalité, d'alliance et de solidarité constamment remis en jeu et / ou réaffirmés.

Ma lecture attentive de l'ouvrage m'a amené à proposer non pas un compte rendu ou une note critique mais une réinterprétation de ses données en me fondant sur deux points essentiels : l'approche anthropologique des récits et celle de la violence sous ses différentes formes.

\section{Généralement Gilsenan présente chaque narration par un synopsis, puis donne en détail son dérouleme (...) \\ 3 Chez les gens du Akkar, les récits narratifs ne sont pas simplement une manière $d$ 'interpréter les $f(\ldots)$}

Pour les récits, je retiendrai la distinction que fait Gilsenan entre ce qu'il appelle narrative, qui est la reconstruction de l'événement, et story, qui est l'événement tel qu'il s'est déroulé (Gilsenan 1996: 163). En effet, dans la narration locale, ce que je traduis donc par récit, les gens ne se contentent pas de raconter, ils ajoutent leurs commentaires. Pour un même événement, les interprétations peuvent être multiples et contradictoires, car elles expriment les tensions existant entre les divers acteurs et groupes sociaux. Ces interprétations entraînent à leur tour des actions violentes ou empêchent l'enchaînement dangereux de la violence 2 . Mais au-delà de cette approche et des réserves que l'on peut avoir sur sa traduction par ce qu'il appelle une « narration anthropologique $3 »$, mon analyse veut insister sur deux points. Tout d'abord, les récits expriment à leur manière l'idée que la prise de risque est constitutive de l'interaction violente ou verbale, que l'aléatoire est inscrit dans l'univers des relations sociales et que toute position acquise est l'objet d'interprétations contradictoires, les unes valorisantes, les autres critiques. En bref raconter, c'est souligner l'ambiguïté des actions violentes dans cette région. En second lieu, l'idée centrale dans la réinterprétation des données de Gilsenan est que l'exercice du pouvoir n'est qu'un aspect exacerbé des rapports entre violence verbale et violence physique qui sont toutes deux inscrites dans toutes les strates de la société.

4 Ce travail fait suite à un premier essai comparatif sur les rapports entre violence physique et usa (...)

5 Gilsenan a choisi de commencer par l'analyse du pouvoir et de son évolution avant de considérer les (...)

Dans un travail publié sur le Rif marocain 4, j'avais souligné la place et le 
rôle de trois catégories d'acteurs sociaux : les jeunes irresponsables, les hommes d'honneur et les hommes de pouvoir. On retrouve ces mêmes catégories au Liban mais, dans ce pays, le contexte social est différent et il faudra souligner la spécificité de cette région du Proche-Orient. On présentera les performances des différents acteurs dans l'ordre suivant : le jeune tueur, le beau parleur, le «leader des jeunes», puis ensuite le chef de famille et I'homme de pouvoir qui est tenté de défier le ciel. Les premiers cherchent à s'imposer par leur action et / ou leur maîtrise de la parole en n'ayant qu'une influence limitée sur leurs agnats alors que les seconds agissent violemment en s'appuyant sur les relations de dépendance et en manifestant leur volonté de pouvoir 5.

\section{Les jeunes dans leurs excès 6}

\section{Destin de tueur •}

Le récit de référence présente une imbrication entre une série d'actions et des interprétations de ces actions par I'opinion locale (Gilsenan 1996 : chap. $x$ 159-188). Il se déroule en trois séquences.

* Première séquence. Deux jeunes amis, un fellah (issu du groupe des paysans cultivateurs) et un agha (du groupe des clients des seigneurs locaux), du même village, emmènent une prostituée sur la plage de la ville de Tripoli. Ils se fâchent entre eux et le fellah blesse d'un coup de couteau le jeune agha. Les deux hommes retournent au village dans leur quartier respectif. L'incident est commenté par les membres du groupe des aghas comme étant une affaire d'honneur entre eux et les paysans, omettant la présence de la prostituée qui a été à l'origine de la dispute. La vengeance s'impose. Dans cette séquence, l'événement est suivi de son interprétation par l'opinion d'une des deux parties, ce qui va entraîner une action.

* Deuxième séquence. Des vieux du lignage prennent l'affaire en main. Ils agissent au nom du lignage dont I'honneur est considéré comme bafoué. L'homme blessé disparaît en tant qu'individu. Les vieux choisissent, parmi les proches de la victime, un jeune homme à peine sorti de l'adolescence, pour agir contre l'agresseur paysan, plutôt qu'un homme marié ayant des enfants. Ils savent qu'avec ce dernier, il leur faudra entretenir sa famille pendant son séjour en prison, alors qu'avec un jeune célibataire ils n'ont pas de souci de ce genre. Mais la suite de l'histoire indique que les choses sont plus complexes. Le jeune homme monte dans le quartier des paysans, va au 
magasin tenu par l'agresseur et le tue. Il redescend tranquillement vers son quartier. Une autre version du récit signale qu'à mi-chemin du retour, il s'aperçoit qu'il a laissé tomber ses babouches dans le magasin du paysan qu'il vient de tuer. Il monte les reprendre et redescend en tournant le dos ostensiblement aux paysans interloqués. L'honneur du groupe est restauré. A l'arrivée du jeune tueur dans son quartier, les aghas disent : «Il est monté [chez les paysans] comme un enfant et il en est redescendu comme un homme » (d'honneur s'entend). Là encore, nous avons une série d'actes qui aboutissent à une vengeance et une opinion qui interprète le meurtre comme une manifestation du sens de I'honneur d'un jeune.

\section{On peut faire I'hypothèse que les paysans ont moins le sens de I'honneur que les aghas et acceptent (...)}

* Troisième séquence. Le tueur est mis en prison. Il y reste plusieurs années. Durant cette période, la paix est rétablie entre les aghas et les paysans. Ces mêmes vieux qui ont provoqué la vengeance proposent à la famille paysanne de la victime le versement d'une compensation. Chaque famille du lignage agha doit y contribuer. Les paysans acceptent cette compensation 7.

Toute l'histoire est par la suite réinterprétée. D'un côté, les paysans proches de la victime indiquent que celui-ci était un fauteur de troubles irresponsable et qu'il est préférable de faire la paix plutôt que de s'engager dans un enchaînement de violence. De l'autre, le jeune tueur agha sorti de prison n'est plus considéré comme un homme d'honneur, mais comme un makhlu', terme qui signifie «sonné », « fêlé ». Ce terme désigne une personne qui n'a plus toutes ses capacités mentales et qui peut provoquer le désordre, le chaos. Cela permet aux aghas de justifier le versement d'une compensation pour réparer l'action d'un irresponsable. Le jeune tueur va être isolé dans son village et ses actes seront considérés dorénavant comme la manifestation de quelqu'un de sonné. Il devient une sorte d'électron libre dangereux qu'il faut isoler.

Ici, il faut prolonger l'analyse de Gilsenan sur un point. La troisième séquence n'ajoute pas simplement des actions et des discours interprétatifs supplémentaires. Elle change la signification des séquence précédentes. Tout se passe comme si la paix n'était possible que si l'on déconsidère, par la parole, les deux responsables (le tueur et la victime) de la violence physique. Alors que, dans les séquences précédentes, l'usage de la parole interprétative provoque ou accompagne une action violente, dans la dernière séquence, elle doit signifier l'arrêt de cette violence. Dans un cas, elle est la continuation de la violence par d'autres moyens, dans l'autre, elle est un moyen de l'empêcher ou de l'arrêter. La violence apparaît ici à la fois comme 
la manifestation de l'honneur et comme la menace du désordre, du chaos.

L'ambiguïté de I'honneur est manifeste dans un autre récit. Un jeune homme marchait dans une rue de Tripoli en compagnie d'un parent lointain. Ce dernier désigna un vieillard marchant devant eux, en disant que celui-ci avait tué quarante ans auparavant l'oncle paternel du jeune homme. Celui-ci sortit un revolver et tua le vieux sur-le-champ. On ne sait pas pourquoi ce parent lointain a provoqué cette action. Il est presque sûr que le jeune homme savait qui était le meurtrier de l'oncle paternel. La vengeance ne s'était pas révélée nécessaire puisque quarante ans étaient passés sans que la famille de la victime n'agisse. Il a suffi qu'une parole soit dite : le meurtrier est devant vous, pour qu'il n'y ait plus d'échappatoire. Le jeune homme qui venge le meurtre de son oncle est emprisonné et quand il sort, on ne parle plus de lui comme d'un homme d'honneur mais comme d'une personne socialement anormale, imprévisible, dont la compagnie est risquée même si on ne va pas jusqu'à dire qu'il est un homme fêlé, un makh/u' (Gilsenan 1996 : 203-205). M. Gilsenan note que le jeune tueur, marginalisé après avoir été un homme d'honneur et très probablement parce qu'il était devenu un homme d'honneur, adopte un comportement étrange comme s'il se conformait à l'image qu'on a de lui. Il remplit des cahiers avec des notes, amasse des magazines, des encyclopédies qui, selon lui, vont expliquer tout ce qui se passe dans l'univers. Cette volonté désespérée de donner sens à tout l'enferme, car il pense que personne ne le comprend, hormis peut-être I'anthropologue (Gilsenan 1996 : 204).

8 Une comparaison avec l'exemple de nomades sarakatsani étudiés par J. Campbell est éclairante. En ef (...)

9 Dans l'autre récit (voir note 5), c'est le compagnon, parent lointain du jeune homme, qui, en désig (...)

10 Cette citation concerne un autre jeune que M. Gilsenan rapproche de celui dont nous parlons, et qui (...)

On ajoutera à l'analyse narrative de Gilsenan la conclusion suivante. Dans le premier récit, les deux acteurs principaux sont les vieux et le jeune tueur. Les premiers agissent au nom du groupe et non en leur nom personnel 8. Ce sont eux qui choisissent le meurtrier, originaire d'une famille pauvre de leur lignage. Il est très probable qu'ils savaient quel serait le destin du jeune tueur. Celui-ci agit mais il est manipulé en quelque sorte, sans pouvoir tirer parti de son action 9. Il est le type même de ce personnage « qui n'a peur de rien, prêt à crier, à défier et à demander des comptes à toute provocation imaginaire ou réelle » (Gilsenan 1996 : 258) 10, il n'a pas la maîtrise de luimême et devient par la suite une sorte d'électron libre imprévisible dans ses 
réactions et difficilement contrôlable. Il ne sait pas parler et réagir de manière appropriée, face à l'opinion. Mais suffit-il de savoir parler pour être un homme d'honneur respecté et respectable?

\section{Le beau parleur •}

Le récit que nous avons relaté ne parle que de jeunes à l'esprit chaud, prompts à agir violemment. M. Gilsenan note que les adultes jugent les capacités des jeunes et leur savoir-faire potentiel de deux manières : « $\mathrm{He}$ might be unskilled into the rhetoric of violence but have the nerve for a killing or he may be rhetorically cunning but have no stomach for a fight » (Gilsenan 1996 : 208). A l'opposé de ces jeunes prompts à agir violemment, il existe donc un autre type de jeune, celui que l'on peut qualifier de beau parleur (Gilsenan 1996 : 206-222). C'est dans le contexte du jaqmara, sorte de combat simulé verbal, qu'il se distingue particulièrement en présence d'un auditoire de jeunes et en prenant généralement pour cible non pas un égal, aussi doué que lui dans la belle parole, mais un jeune plus faible que lui dans ce domaine du savoir parler mais qui manifeste des ambitions sur ce plan. On notera qu'on ne s'attaque pas à un très proche parent (un frère par exemple), ni à un étranger que l'on ne connaît pas. On évite aussi de se mesurer à une personne d'une autre génération. Le jeu doit se dérouler en principe entre jeunes qui se connaissent et qui se fréquentent régulièrement. La performance verbale doit être flamboyante pour être appréciée. Le jeu consiste à prétendre avoir une compétence supérieure à celle de son opposant sur un sujet choisi. L'audience sait qu'il n'en est rien, de même que le beau parleur. Celui-ci doit toujours maintenir une distance entre sa personne et sa performance. A l'opposé, il doit déstabiliser son opposant en le poussant à bout, en l'amenant à se mettre en colère et à perdre le contrôle de lui-même (mithammis), c'est-à-dire à prendre au sérieux ce qui n'est après tout qu'un jeu, un amusement (mazah), ce que l'audience ou le beau parleur ne manqueront pas de lui rappeler. Gilsenan raconte différentes confrontations de ce genre. Le premier exemple est celui de ce beau parleur qui ne connaissait rien à la mécanique des voitures et qui en provoque un autre qui avait trois ans d'expérience à ce sujet. C'est en prétendant tout connaître mieux que le jeune mécanicien, et en le bombardant de questions prétendument techniques qu'il arrive à le mettre en colère et donc à le déstabiliser. En principe, il ne doit pas y avoir d'enjeu en dehors du combat simulé lui-même. Comme cela est un jeu, un amusement, il ne faut pas qu'il vienne après un conflit réel ou qu'il ait pour objet un travail, etc. Ce même jeune mécanicien reçut un jour un coup de téléphone venu disait-on de la capitale pour du travail urgent. Il partit vite, malgré les avertissements selon lesquels il pouvait s'agir d'un gros mensonge. Il revint le lendemain, furieux et décontenancé. Il avait perdu deux journées de travail. Le beau parleur, qui l'avait déjà pris pour cible, fut accusé d'être derrière cette mauvaise 
blague. On le critiqua parce qu'il avait dépassé les limites du jeu et avait fait perdre du temps à ce pauvre mécanicien crédule. Le mazah (amusement) avait empiété sur le maslaha (le travail qui fait vivre une famille) (Gilsenan 1996 : 221-222).

Gilsenan raconte, d'autre part, une sorte de joute entre deux cousins à propos des élections nationales. Il s'agissait de savoir si l'oncle maternel de Tariq allait voter pour le père d'Ahmad. Tariq dit qu'il allait soutenir un autre candidat et que son oncle le suivrait. Tout était prêt pour le jaqmara, le combat simulé verbal : le public était présent, la campagne électorale avait commencé. Tariq était le beau parleur par excellence et sa rhétorique était telle qu'il arrivait à retourner tous les arguments d'Ahmad contre lui. Plus encore, il ne cessait de montrer à l'auditoire comment il pouvait jouer avec ce qui est sérieux (Gilsenan 1996 : 217) et contrôler la situation autant que lui-même. Son rival Ahmad commença à perdre patience et, dans un état d'irritation, il cria : «Nous sommes la famille de... Et ton oncle maternel embrasse la main de notre père » (ibid. : 217). La confrontation se déplaçait et devenait sérieuse, puisque la réputation des deux familles était en question. Tariq trouva la parade en adoptant dans sa réponse un ton sarcastique : « C'est vrai qu'il embrasse sa main, mais c'est par respect (ihtiram) et rien d'autre. Mais quand il s'agit des élections, le respect ne compte pas » (Gilsenan 1996 : 218). Ahmad, rendu encore plus furieux, se mit à hurler et se fit menaçant. Tariq, gardant son calme, répondit : «Ghalat» (c'est faux, c'est une erreur). Cela signifiait que son vis-à-vis ne jouait pas comme il fallait et que lui, Tariq, ne se laisserait pas entraîner dans une confrontation sérieuse. Ici, à la différence du pur amusement, il y a un enjeu (le vote pour une élection), mais le beau parleur veut faire en sorte qu'il s'agisse d'un combat simulé verbal. A chaque fois que le jeu risque de déraper, il arrive à trouver la parade, à ne pas se laisser entraîner. On voit comment savoir parler est une manière d'humilier l'adversaire qu'on choisit, sans avoir à passer à l'action violente. L'opinion publique villageoise est certes admirative de sa maîtrise de la belle parole, mais elle considère aussi ce virtuose du verbe comme un fattan, quelqu'un qui peut semer la zizanie. Par ailleurs, on le soupçonne d'être un jaban, un lâche qui reculera quand il s'agira de se battre pour son honneur.

En bref, on peut dire que le jeune tueur ne sait pas parler et que le beau parleur ne sait pas agir. Aucun des deux n'a une maîtrise à la fois du jeu verbal et de la violence physique. De plus, aucun n'a le prestige et l'autorité dans le groupe des aghas.

\section{Le « leader des jeunes » ou sheikh ash-shebab •}


En dehors des jeunes violents et des beaux parleurs, il existe parmi les aghas des personnages qui sont appelés par le terme de sheikh ash-shebab que I'on peut traduire en première approximation par «leader des jeunes ». Gilsenan désigne ce type d'acteur par la locution leader of the fighting men. Si l'on suit la description qu'il en fait dans la fin de son ouvrage, c'est le terme «leader des jeunes bagarreurs » (plutôt que combattants) qui paraît adéquat, car ce personnage est en quelque sorte le chef des têtes brûlées comme on le verra plus loin. Le leader dont il est souvent question est prénommé Hassan. Celui-ci a réussi à acheter d'occasion plusieurs camions pour le transport, la fabrication du ciment, etc. Avec ses véhicules, il obtient des contrats en ville pour la construction de bâtiments ou de pistes d'aéroport, la réfection des chaussées, etc. Grâce à cela, il peut fournir du travail, plus ou moins régulier, à de jeunes agnats de son segment de lignage qu'il essaie de fidéliser pour en faire ses dépendants (Gilsenan 1996 : 293-295). Le titre de sheikh ash-shebab ou «leader des jeunes » est à la fois un titre honorifique et une manière un peu ironique de le dénommer, nous dit dans une note Gilsenan (Gilsenan 1996 : note 11 : 359-360). En effet, en plus d'une certaine efficacité économique, trouver du travail à ses proches, il est connu pour sa promptitude à réagir dans les affaires d'honneur concernant son segment de lignage. Deux événements dans lesquels il est intervenu sont relatés par Gilsenan.

Dans le premier, la scène se passe au village (Gilsenan 1996 : 292-293). Un jeune se précipite dans son atelier où Hassan se trouve en compagnie d'ouvriers, en criant : «Nos femmes se font violer, le sang est répandu dans le village [sous-entendu par la police], et vous êtes assis là sans réagir! » Tout le monde comprend que la police arrive au village et que tout le reste n'est qu'invention. Hassan se précipite dehors, le fusil à la main, à la rencontre du préfet et de la police qui veulent agir contre ceux qui ont coupé la route de la montagne. Il hurle son nom : « Je suis Hassan Abd es Salam » face au préfet qui s'avance sans arme. Les jeunes se tiennent sur les toits, les collines, prenant pour cible la police. Le préfet reste calme et se contente de dire: «C'est honteux, c'est honteux » comme s'il voulait montrer sa supériorité en refusant la confrontation en termes d'honneur. Le notable agha (voir plus loin à son sujet) et les vieux s'avancent vers le préfet pour le recevoir et pour négocier avec lui. Ils demandent aux jeunes de rentrer chez eux, ce qu'ils font. Le sheikh ash-shebab se retire comme les autres.

Il faut noter que l'intervention du jeune avait tout d'une fausse nouvelle et qu'il s'agissait de provoquer une réaction extrême du sheikh ash-shebab. Celui-ci agit de manière précipitée et irréfléchie, et ensuite il doit laisser la place à des aînés. Cela ne signifie-t-il pas que son comportement participe de cette irresponsabilité des jeunes qui sont avec lui ?

Le second récit est plus complexe (Gilsenan 1996 : 269-280). Dans Tripoli, 
un chauffeur de bus scolaire originaire du village a une altercation avec un importun. De retour chez lui, il raconte l'incident. Des membres de son segment de lignage, conduits par le «chef des jeunes», décident de mener une expédition punitive contre le responsable. Il s'agit plus de faire une démonstration de force que de s'engager dans une bataille violente, mais comme on ne peut prévoir comment les choses vont tourner, ils emportent des fusils et des bâtons. Deux heures après ils reviennent et le sheikh ashshebab raconte ce qu'ils ont fait. Au dépôt des bus en ville, ils ont rencontré les collègues de leur parent agressé, mais pas l'agresseur qui n'était pas présent. Au cours de la discussion, un jeune de l'expédition voulut prendre à partie les chauffeurs de bus. Le chef des jeunes, saisissant son fusil, tira avec violence en arrière le jeune à l'esprit échauffé et ramena son groupe au village. Personne n'osa contester en sa présence la narration du sheikh ashshebab. Au contraire on approuva son action et notamment la manière dont il empêcha le jeune à l'esprit échauffé de provoquer une bagarre violente. Mais dans les jours qui suivirent, les discussions commencèrent et de multiples interprétations furent avancées par les uns et les autres, soulignant l'ambiguïté de la situation. Est-ce que le « leader des jeunes » avait bien agi en se précipitant à la ville avec ses hommes? N'aurait-il pas mieux valu attendre et réfléchir sur la manière la plus appropriée d'agir ? Fallait-il emporter les fusils ou seulement des canifs ? Pourquoi aller au dépôt des bus et s'en prendre aux chauffeurs au lieu de rechercher la maison et les proches du responsable de l'incident ? Qu'est-ce qu'une démonstration de force qui n'atteint pas celui à qui elle est destinée ? Et le chauffeur agressé n'était-il pas lui aussi une sorte d'irresponsable qui avait provoqué bêtement I'incident ? Pouvait-on risquer de perdre son emploi pour si peu de chose ? Les membres du segment opposé à celui du sheikh ash-shebab se moquèrent du comportement irresponsable de tout le groupe des jeunes dans ces circonstances.

En bref, le « leader des jeunes », tout en étant respecté en apparence, est considéré, par des allusions, comme n'ayant pas toujours les réactions appropriées aux événements. Certes il n'est pas comme ces jeunes à l'esprit échauffé qui ne savent pas se contrôler. Mais au niveau où il se place - il est un homme qui a des moyens et des dépendants - il ne maîtrise pas totalement la situation. Il sait provoquer le préfet et montrer qu'il ne laissera pas la police faire ce qu'elle veut au village, il évite le pire lors de l'expédition des jeunes au dépôt des bus, mais, dans les deux cas, il agit avec précipitation et un doute s'installe quant à ses capacités à diriger son groupe. Il est le sheikh ash-shebab, le «leader des jeunes», mais il n'est pas le qabaday, I'« homme fort » le chef de famille. Ce point de vue est signalé de manière allusive par Gilsenan mais n'est pas clairement exprimé. Dans aucune partie de l'ouvrage, il ne regroupe les données concernant les différent types de jeunes comme nous avons essayé de le faire. 


\section{La recherche de prestige et de pouvoir}

\section{Le qabaday, l'« homme fort »}

Trois facteurs sont déterminants pour la compréhension de la position de ce personnage : la possession de la terre, le sens de l'honneur et les rapports complexes avec le seigneur. Par ailleurs, il faut distinguer entre les hommes du passé dont parle la mémoire généalogique et les hommes du présent qu'a connus l'anthropologue. Il faudra parler des premiers avant d'en arriver aux seconds. Deux récits vont nous servir de guides pour comprendre ces histoires de qabaday, $d^{\prime} \ll$ homme fort $»$.

Le premier est relaté dans le dernier chapitre de l'ouvrage (Gilsenan 1996 : 306-309). On y parle d'Abd ar-Rahman Said qui fut, dit-on, la figure exemplaire de ce que doit être un agha. Sa réputation était inégalée de même que son courage et son sens de l'honneur. Il fut le plus proche et le plus estimé des compagnons (murafiq) du seigneur Abboud bey qu'il servit le plus fidèlement possible. Il fut efficace quand il fallait affronter les ennemis de son patron, contrôler les travailleurs sur ses terres, ou maintenir et renforcer le prestige du nom du bey. Celui-ci, pour le remercier et pour I'honorer, lui donna dix hectares d'oliviers et, à d'autres occasions, de I'or. Selon la légende, il ne s'agissait pas d'un paiement, mais d'un témoignage de respect, de la reconnaissance de l'excellence de cet homme. Celui-ci n'avait pas besoin de faire garder ses terres contre les voleurs car son seul nom suffisait. En bref, Abd ar-Rahman Said fut cet homme d'honneur qui a su accumuler des terres et non les perdre, qui fit de sa fidélité à son maître une marque de sa grandeur. La suite de l'histoire est beaucoup moins édifiante. A sa mort, au début de la Deuxième Guerre mondiale, cet homme laissa un seul héritier, un enfant en bas âge appelé Abu Abduh. Quand celui-ci devint adolescent, il commença à dilapider son héritage par des dépenses ostentatoires dans les deux villes de Tripoli et de Beyrouth, en compagnie des jeunes de son village. Toute la fortune qu'avait amassée son père $y$ passa et Abu Abduh se retrouva ruiné et sans personne pour l'aider. Après un travail dans la police et un séjour au Koweït, il se retrouva homme de main d'un seigneur pour lequel il mena des actions hasardeuses. Recherché par la police, il vit en hors-la-loi et joue le bouffon, l'amuseur public. Cette histoire raconte donc comment un fils eut le comportement exactement opposé à celui de son père, détruisant tout ce qu'avait construit ce dernier. Si son père était un compagnon respecté du seigneur Abboud, Abu Abduh est un pauvre hère manipulé par un autre seigneur. Il faut noter que, tout en reconnaissant son manque de caractère, Abu Abduh accuse ses agnats de ne 
pas l'avoir aidé mais au contraire de l'avoir poussé à dépenser. En bref, c'est grâce à un seigneur que le père avait construit sa réputation et sa fortune, et c'est poussé par ses agnats qu'un jeune homme insouciant détruit son héritage et son honneur.

L'autre récit du chapitre viii est plus complexe, mais l'on y retrouve les mêmes éléments (Gilsenan 1996 : 115-139). La première version du récit est racontée à l'anthropologue par le maire du village. L'affaire a lieu durant la Première Guerre mondiale, période durant laquelle il y avait la famine. Le grand-père du maire, un agha d'un certain renom, cheminait en compagnie d'un important seigneur du lieu. Celui-ci mangeait une orange et l'agha dit : « Je donnerais n'importe quoi pour une orange. » Le seigneur lui dit : «Me donnerais-tu ce terrain d'oliviers ? - Oui », répondit l'autre. Le bey lui donna le fruit. Plus tard il lui rappela sa promesse et l'agha lui répondit avec superbe : «Prenez-le » (le terrain). Pour le maire, cette histoire est symptomatique du comportement de son grand-père, grand propriétaire terrien qui perdit progressivement toutes ses terres au profit des seigneurs et il ne reconnaît pas de l'honneur à celui qui a sacrifié ses propriétés. Une autre interprétation de ce geste, suggérée par l'anthropologue, est qu'il s'agit bien d'un homme d'honneur, car il sait tenir la parole donnée et il n'hésite pas à perdre son bien le plus précieux pour acquérir, par un mot superbe, un prestige, une réputation, un nom. A la différence de l'autre récit, avoir un comportement d'homme d'honneur ne va pas avec la possession de la terre mais avec sa perte. De plus, le bey n'agit pas en faveur de l'agha en renforçant son autorité et sa richesse comme pour Abd ar-Rahman Said mais cherche à le piéger pour l'affaiblir. Il utilisa son sens de l'honneur pour le tromper et l'amener à vendre ses biens.

Il est dommage que Gilsenan ne mette jamais en parallèle les deux récits. Je voudrais, en conclusion de cette section, les comparer. A première vue, ces deux récits semblent se contredire. En fait, ils montrent bien que la relation de dépendance entre un seigneur et son client est un combat permanent. Dans un exemple, c'est l'agha qui oblige en quelque sorte son bey à payer au prix fort sa fidélité et son action en sa faveur. Il lui démontre que son nom, sa réputation d'homme de pouvoir dépendent de son client et le seigneur n'a pas d'autre choix que de lui donner ses terres. Plus encore, il marque avec force que son honneur est fonction du caractère exemplaire de sa fidélité à son patron, le don de la terre en étant le signe éclatant. Dans l'autre exemple, l'agha ne maîtrise pas la situation mais la subit. C'est son nom, sa réputation qu'il veut promouvoir et non ceux du bey. Il n'est plus question de fidélité à un supérieur comme marque de son honneur. Le bey agit et réussit à affaiblir celui qui a montré ainsi les signes d'une certaine fatuité. En bref, la relation de dépendance apparaît à la fois comme une forme 
« d'attachement », de fidélité, de négociation et de rapport de forces.

Mais ces deux récits sont semblables sur un point : ils se concluent par une ruine. Dans le premier cas, c'est le fils qui dilapide la richesse amassée par son père, dans le second cas, c'est l'homme de prestige qui se détruit luimême. Il apparaît donc que le pouvoir d'un agha est précaire et finit par être détruit soit par ses « frères », soit par son supérieur.

Il est intéressant de continuer l'histoire de l'agha qui vendit sa terre pour une orange. Gilsenan raconte que son fils, le père du maire, commença sa carrière sans argent, sans héritage. Il réussit à acquérir des terres au détriment de ses agnats. Dans la terminologie locale, on dit qu'il les « mangea ». Il faut noter que son segment de lignage était très affaibli par des conflits meurtriers entre demi-frères et entre cousins. Il profita de cette situation pour devenir le ra'is el aila, le « chef de famille » de son segment de lignage. Un agha acquiert de la richesse terrienne non seulement par l'intermédiaire d'un bey mais en captant les terres de ses proches, de ses égaux. De la même manière, un agha est affaibli aussi bien par un bey que par ses agnats.

\section{La volonté de pouvoir chez les seigneurs •}

Ils se disent d'origine turque, formant un seul groupe lignager dont l'ancêtre fondateur est supposé être venu s'installer dans la région au xviiie siècle. Ils se sont imposés dans les différents villages du Akkar comme grands propriétaires fonciers et ont été nommés beys ou pachas, administrateurs de la région par l'autorité ottomane. Leur rôle était de lever les impôts pour I'Etat et de contrôler militairement leur domaine. Ils habitaient la montagne, s'opposaient entre eux pour étendre leur domaine aux dépens de leurs rivaux de même statut ou au contraire pour limiter l'action prédatrice de ces derniers. La disparition de l'Empire ottoman n'a pas signifié la fin de leur pouvoir. Certains vont établir des liens étroits avec l'autorité coloniale française pour asseoir et étendre leur pouvoir. Mais un double changement va s'opérer (cf. Gilsenan 1996 : chap. vi 79-94). Tout d'abord, certains beys vont profiter du mandat français pour moderniser leur agriculture, utilisant les nouveaux moyens techniques pour augmenter et varier leur production et pour la vendre sur le marché national. Ensuite ils vont s'installer en ville, y acheter une demeure sans vendre celle qu'ils possèdent dans la montagne. Ce mouvement concerne aussi la plupart des grandes familles maronites et certains druzes. C'est en milieu urbain qu'ils élargiront leur activité économique et politique. Ils profiteront de nouvelles facilités pour s'engager 
dans le grand commerce, dans la spéculation immobilière et / ou financière. Ils vont aussi participer au jeu politique national qui commence à prendre forme, en devenant députés ou ministres, en ayant leurs entrées dans les différentes administrations et en devenant des intermédiaires pour aider leurs clients de la montagne. Ils développent un style de vie urbain tout en gardant une œil vigilant sur leur domaine de la montagne grâce à leur régisseur agha dont le rôle va devenir de plus en plus important. L'indépendance du Liban en 1945 va accentuer ce mouvement (cf. Gilsenan 1996: chap. vii 95-111). La mise en place d'un parlement, d'un gouvernement et d'une administration en principe régie par des règles démocratiques ne modifiera pas pour l'essentiel le système économicopolitique que le pouvoir colonial avait établi. Les seigneurs de la montagne continueront leur activité économique urbaine sans laisser tomber leur domaine agricole. Avec les chefs issus de la ville, ils entrent dans l'arène politique en s'appuyant sur leurs clients traditionnels. Les rivalités et les conflits locaux entre seigneurs de la montagne vont se répercuter au niveau national. Nous en verrons un exemple particulièrement significatif. Le grand changement qu'apporte l'indépendance par rapport aux périodes précédentes, c'est qu'il n'y a plus d'autorité extérieure pour limiter le jeu politique local et national. L'Etat libanais devient l'arène où s'exercent toutes les alliances et les oppositions entre les différents chefs politiques s'appuyant sur un électorat qui forme l'essentiel de leur clientèle. Un homme politique est un patron qui trouvera un emploi à ses clients, leur facilitera les relations avec l'Administration, les sortira de prison ou résoudra leurs problèmes judiciaires, etc. Il est aussi un homme puissant économiquement qui utilisera ses fonctions politiques, son réseau d'alliances pour accroître, par différents moyens légaux ou non, ses ressources et sa richesse. Cela signifie aussi que la perte d'influence politique peut aussi amener un certain appauvrissement. Il serait faux de penser que ces hommes politiques contrôlent tous les leviers de l'économie nationale et locale. D'autres couches sociales, notamment des commerçants, des financiers ou des industriels, disposent de moyens plus ou moins importants mais, en règle générale, il leur faut passer par ces hommes de pouvoir pour s'enrichir. Le système politique libanais postcolonial a cette caractéristique majeure qu'il est composé à tous les niveaux de patrons qui s'allient ou s'opposent au gré des circonstances. Il n'y a aucune autorité reconnue qui transcende ce jeu ou qui ait réussi à s'imposer pour devenir le symbole de l'unité nationale.

Pour revenir à la région du Akkar, les beys se signalent par le pouvoir effectif qu'ils possèdent ou qu'ils ont possédé jusqu'à une époque récente et qui les situe dans la logique que nous venons de décrire. Ils existent aussi par la mise en scène très codifiée par laquelle ils donnent à voir ce pouvoir, par les représentations dont ils sont l'objet et enfin par leur rapport à la mort sous 
différentes formes. On examinera successivement ces deux points.

\section{a) Le théâtre du pouvoir}

M. Gilsenan reconstitue dans le premier chapitre la figure de Mohammed Abboud, fils d'un grand bey, Abboud ar-Razzaq (Gilsenan 1996 : 3-22). C'est l'été 1953, à la veille des élections législatives auxquelles se présente ce jeune seigneur. Habitant généralement en ville, il a fait construire, dans son village d'origine, une grande villa sur le flanc de colline surplombant les quartiers aghas. Il y reçoit ses clients pour discuter des affaires concernant ses terres et la campagne électorale ; il y accueille les invités distingués dont il espère l'appui. D'ordinaire, les maisons locales ont un mur aveugle sur la rue, la porte d'entrée se situant à l'arrière de la demeure. La villa possède un grand balcon et une grande porte d'entrée sur le devant. Le bey vient s'asseoir sur ce balcon. Il est là pour voir son village et pour être vu et même pour défier ses rivaux qui vivent dans d'autres demeures sur d'autres collines. Tout ici tient d'une forme de ritualisation du pouvoir. A côté du bey se tient son murifiq, son «compagnon», un agha qui est son homme de confiance. Celui-ci parle avec une voix forte et des gestes larges. Il a I'habitude, nous dit M. Gilsenan, « de donner des ordres, de surveiller les paysans, de confronter les défis des rivaux, de chasser seul dans la montagne » (Gilsenan 1996 : 4). Il correspond au portrait que nous avons dressé plus haut des qabaday. Son seigneur parle avec une voix plus douce, proche de celle des personnes éduquées de la haute bourgeoisie. Un qahwiji, un préparateur de café, un nargalji, préparateur de la pipe à eau, et d'autres clients aghas à portée de voix complètent la scène. Le bey utilise ici le narguilé (alors qu'en ville, il fume le cigare), signe de son statut. Dans les occasions extraordinaires, il fera sortir pour les invités de marque plusieurs pipes à eau avec des vases en cristal de Bohême ou de Damas, magnifiquement décorés. Chaque narguilé est un objet de prestige qui a une histoire et sa généalogie. On peut raconter comment il a circulé de bey à bey, jusqu'à arriver chez celui qui le détient et qui le présente à un public choisi. Le seigneur fait jouer dans sa main un rosaire. Il en a plusieurs et comme les pipes à eau, certains, faits avec des pierres semi-précieuses, ont une généalogie. Tout semble fait pour que le pouvoir se manifeste dans sa grandeur, sa splendeur, avec une sorte de force tranquille.

Il est très probable que dans ce lieu, durant ces moments, il y a un protocole très élaboré pour prendre la parole selon son rang, des usages à respecter dans les discussions, dans les doléances, les réprimandes des fautifs, les décisions. Comme toute mise en scène dans cette région, savoir parler est aussi une manière de continuer la violence sous d'autres formes. On est dans ces moments où le pouvoir doit se montrer «à son aise », où les rivalités se 
donnent à voir et s'expriment de manière feutrée. Il s'agit d'œuvrer pour échapper au cycle de la violence ou au contraire pour préparer des meurtres futurs. Malheureusement, Gilsenan n'a probablement pas assisté à ces séances qui doivent se dérouler dans le grand salon de cette villa et ne peut nous en donner une description détaillée.

\section{b) La longue attente de la vengeance}

Le chapitre ii nous donne une autre vision de cette villa et de son propriétaire (Gilsenan 1996 : 23-37). A son arrivée sur le terrain en 1971, l'anthropologue est emmené par un groupe de jeunes aghas et un jeune fellah visiter cette villa. Celle-ci est à I'abandon, sans porte, ni fenêtre. A l'intérieur, une seule chambre est fermée à clé, c'est celle dans laquelle se trouve le cercueil de son propriétaire, Mohammed Abboud, le même personnage dont il a été question précédemment. Le récit de son assassinat est particulièrement intéressant. On est toujours en 1953. Les élections législatives se préparent. Mohammed Abboud, qui avait été dans le passé député puis ministre, et qui avait été battu aux élections par son rival, se représentait de nouveau et constituait une menace pour le député sortant. Celui-ci était soutenu par le nouveau président de la République libanaise qui convoqua dans son palais les deux hommes. Il s'agit de persuader Mohammed Abboud de se retirer. Un tueur, homme de main de son rival, était à l'affût à la porte du palais. Si les deux rivaux sortaient ensemble, c'est que Mohammed Abboud avait accepté la proposition du président, et il fallait le laisser partir. Mais Mohammed Abboud se présenta seul, signe qu'aucun accord n'avait été trouvé. Il fut assassiné sur les marches du palais. Son père, le grand bey Abboud ar-Razzaq, refusa que son fils soit enterré jusqu'à ce qu'à ce que la vengeance soit accomplie. Le cercueil recouvert d'un drapeau libanais fut placé dans cette chambre. Dix-huit ans après, durant le séjour de l'anthropologue en 1971, il y était encore. Le comportement du père indique de manière forte que son honneur est souillé, qu'il n'y a pas de chance pour que l'histoire du meurtre soit oubliée ou qu'il y ait un pardon. Comme son fils était mort sans descendance, Abboud épousa malgré son âge avancé une femme agha dont il eut un fils qu'il nomma Mohammed comme son demi-frère assassiné. Pour sa protection, il fut élevé à l'étranger en attendant qu'il revienne venger le mort.

Ici je prolongerai l'analyse de Gilsenan en insistant sur un point qui n'a pas retenu l'attention de l'auteur. La décision du bey de ne pas enterrer son fils contredit la loi religieuse musulmane. Selon cette loi, tout mort doit être enterré rapidement, si possible dans les vingt-quatre heures. Généralement dans les affaires d'honneur, on enterre la victime, mais les rites funéraires ne 
sont terminés que lorsque le mort est vengé. Par son acte, Abboud montre de manière ostentatoire qu'il place son honneur au-dessus de la loi religieuse, sans que personne n'ait trouvé, semble-t-il, rien à redire. Ce geste est à la fois une manifestation du sens de l'honneur et une sorte de défi au ciel.

11 Certes tous les beys ne vont pas jusqu'à cet extrême, mais il n'est pas rare que la mémoire locale $(. .$.

M. Gilsenan nous présente ce personnage d'Abboud comme l'exemple le plus extrême du pouvoir des beys. Il profita de toutes les occasions pour développer une agriculture commerciale performante. Il utilisa ses relations avec les autorités coloniales françaises pour accroître et affirmer son influence politique. Il poussa son fils à se présenter aux élections et à devenir ministre. Mais ce qu'on raconte à son propos va plus loin. Homme cruel (zalim), violent, il n'hésite pas à tuer un paysan pour essayer un nouveau fusil, à fracasser le crâne d'un enfant contre un mur parce qu'il pense que ce dernier lui a manqué de respect (Gilsenan 1996 : 8). "Il crée les événements et agit sur les autres comme il le veut, du moins c'est ainsi que les figures du pouvoir sont imaginées et ainsi que les hommes en parlent. Ils respectent Abboud. Ils le craignent » (ibid.). En bref on pense que le pouvoir est arbitraire et se veut absolu, sans limites. Mais il y a plus. Abboud ar-Razzaq fit construire une grande mosquée en haut d'une colline surplombant les cimetières des beys. Dans l'enceinte, il plaça ce qui devait être sa future tombe qui prit la forme de celle d'un saint. Ce «mausolée de son pouvoir temporel » (Gilsenan 1996 : 20) le mettait à part et au-dessus des autres grands seigneurs. Effectivement après sa mort Abboud ar-Razzaq fut enterré comme il l'avait prévu. Après le défi au ciel, c'est la sacralisation de sa mort par ses propres moyens qui est offerte au public et non une élection divine 11.

\section{La politique comme mise en scène de la violence}

Au terme de cette analyse, plusieurs points méritent d'être soulignés : de bas en haut de l'échelle sociale, les relations sociopolitiques se déroulent comme un spectacle, comme une performance théâtrale. Le jeune tueur agha ne se contente pas de tuer, il le fait avec panache et inconscience, n'hésitant pas, après son acte, à remonter chez sa victime pour récupérer ses babouches. Le beau parleur n'existe pas sans la comédie qu'il met en scène pour s'amuser aux dépens de son partenaire de jeu. Le «leader des 
jeunes » fait face à la police avec son fusil en menaçant de faire parler la poudre. Le bey installe le cercueil de son fils assassiné dans sa villa, pour donner à voir de manière spectaculaire la décision qu'il a prise de ne l'enterrer qu'après la vengeance, etc. Chacun dans sa position joue et le public interprète l'action qui se déroule devant lui. Tout en appréciant à sa juste mesure le spectacle, ce public ne se prive pas de semer le doute par sa parole, ses discours, ses interprétations, ses rumeurs, sur la performance des acteurs : le jeune tueur est un « fêlé », le beau parleur est probablement un lâche, le «leader des jeunes » ne sait pas apprécier à sa juste mesure les incidents qu'il affronte, le seigneur garde la marque de sa souillure, etc.

Mais au-delà de cet aspect baroque de la violence à la fois réelle et ritualisée, on doit souligner que :

- les aghas ont plusieurs personnages différents qui agissent : les vieux qui se mobilisent pour le lignage, les hommes violents, les beaux parleurs, le «leader des jeunes » et les « hommes forts». Au contraire, le groupe des seigneurs n'existe que par ces hommes de pouvoir qui s'affrontent ou s'allient, les autres devant s'effacer derrière eux ;

- le qabaday agha peut être considéré à la fois comme un homme d'honneur et comme un «homme fort». A la différence des jeunes de son groupe, il possède des terres et impose son autorité sur son groupe. Toute sa vie, il doit forcer le respect du seigneur dont il dépend et à qui il doit être fidèle. La subordination à un bey n'est valorisée que par une attention et un combat permanents entre les deux partenaires. Sans la force de l'agha, le pouvoir des beys n'existe pas. Sans le bey, l'agha ne peut s'imposer comme « homme fort». Si l'un faiblit, l'autre prendra avantage, la relation sera fragilisée et la dépendance se transformera en un simple affrontement. La relation entre les deux personnages doit s'établir sur la longue durée mais elle est semée d'embûches et en danger de rupture ;

- si l'agha doit en principe servir son bey, ce dernier ne sert personne. Il n'agit pas pour défendre une cause. Son pouvoir n'a pas d'autre finalité que sa propre autoglorification. Aucune autorité religieuse reconnue ne limite son action. Au contraire, il peut se permettre de contrevenir à la loi musulmane sans qu'il subisse de sanctions ;

- dans toutes ces relations et ces affrontements, il y a les parties qui s'opposent ou s'allient, mais il y a aussi l'opinion, diverse, qui se manifeste et 
agit par son interprétation des événements, par les rumeurs qu'elle véhicule, par l'action qu'elle provoque pour lancer la violence ou l'interrompre. C'est elle qui rend tout équilibre fragile en introduisant le doute sur les uns ou les autres. C'est elle qui fait agir le tueur et le transforme par la suite en homme « sonné », c'est encore elle qui souligne combien le beau parleur cache un lâche, c'est elle qui sème le doute sur le comportement du «leader des jeunes », etc. ;

- actuellement, l'agriculture n'est plus la seule activité qu'exercent ces villageois qu'ils soient aghas ou fellahs. Ils deviennent chauffeurs (de camions, de bus, de tracteurs, de taxis) ou mécaniciens, entrent dans l'armée, ou vont en ville pour y travailler dans le commerce ou sur les chantiers. Les beys ne sont plus les détenteurs de la plus grande partie des terres dans les villages de montagne. Installés dans les villes, ils prennent les habitudes de la haute bourgeoisie du monde urbain et leurs fils semblent se désintéresser du monde villageois. Dans cette région, on bascule de l'univers centré sur le jeu de la violence et le rapport de pouvoir à celui où la priorité est la recherche de l'emploi, du travail et donc de l'intérêt économique dans un marché national. Peut-on dire pour autant, comme semble le faire Gilsenan, que toute cette mise en scène, ce « cinéma », ce donner à voir que les uns et les autres font, n'est plus qu'un jeu dérisoire, un héritage des illusions passées dont ils n'arrivent pas à se débarrasser ? Je ne le pense pas. Dans le passé comme à la veille de la guerre civile, on est dans un univers qui associe étroitement les rapports politiques et économiques. Les aghas éminents du passé ne pouvaient s'imposer uniquement par leur sens de I'honneur, il leur fallait avoir une richesse en terre leur permettant d'affirmer leur prestige et leur pouvoir. Certains ont tout perdu parce qu'ils ont privilégié le jeu de l'honneur au détriment de la possession d'un vaste domaine. Les aghas du présent qui veulent s'affirmer sont obligés, comme le montrent de nombreux exemples, de jouer sur les deux registres de l'ambition politique et de la richesse économique. On peut dire la même chose pour les beys. Même installés en ville, même engagés dans des aventures économiques, il leur faut rechercher un pouvoir politique, garder leurs clients de la montagne pour se faire élire au parlement, etc. La situation s'est donc complexifiée, de locale, elle est devenue nationale, mais elle reste marquée par le jeu des passions politiques ;

- comme je l'ai souligné dans l'introduction, on retrouve les mêmes catégories sociales (les jeunes, les hommes d'honneur et les hommes de pouvoir) dans le Rif marocain, bien que le contexte soit différent, de même que le rapport entre violence physique et violence verbale. Plutôt que de comparer les deux univers sociaux, je voudrais insister ici sur une différence majeure entre les deux pays. Au Maroc, il y a des médiateurs locaux, 
hommes de la baraka, négociateurs infatigables, respectés et parfois redoutés, qui savent arrêter la violence au nom de Dieu quand elle risque de tourner au massacre. Par ailleurs le roi, placé à la tête de la communauté marocaine, est aussi issu d'un groupe de saints. Désigné comme le Commandeur des croyants, il est le seul à posséder à la fois le pouvoir politique et l'autorité religieuse qui lui est conférée par la bénédiction divine, la baraka.

12 Certes la situation chez les sunnites du Akkar n'est pas celle des maronites, des druzes ou des chi (...)

Au contraire au Liban, tout se passe comme si l'action politique était prééminente sans le secours, le contrôle ou la légitimation de la religion. Il n'y a pas de saints qui jouent les médiateurs. Ce sont les acteurs politiques qui doivent trouver par eux-mêmes les chemins de la paix. Aucun homme politique, qu'il se situe au niveau local ou national, ne peut se réclamer d'une sacralité quelconque. L'autorité religieuse qui transcende les divisions, les factions, qui organise un Etat, n'existe pas. Le Akkar et plus généralement le Liban vit le politique comme un univers profane qui ne se légitime pas par une quelconque autorité ou valeur religieuse 12 et cela peut expliquer en partie la nature de la guerre civile qui a ravagé le Liban entre 1975 et 1990.

13 Il me paraît faux de penser que la guerre civile libanaise se ramène à un affrontement entre commun (...)

On a beaucoup glosé pour expliquer cette guerre. On a cherché les coupables (les Palestiniens, la droite maronite, la gauche musulmane, les Syriens, les Israéliens, les hommes politiques, etc.), comme s'il suffisait de dénoncer des responsables et de s'apitoyer sur les victimes. La situation sociale et politique ne peut se réduire à ces schémas simplistes. On n'entrera pas dans un débat sur ce sujet car on est encore loin de disposer des éléments qui permettraient de comprendre ce qui s'est passé. A juste titre, Gilsenan ne prétend pas chercher les raisons de cette violence aveugle. Mais son analyse est là pour nous dire sur quel terrain miné cette guerre civile s'est déclenchée. Il nous montre avec force comment le jeu politique se faisait au bord de l'abîme. Certes, il y avait des moyens de modérer cet échange, pour empêcher l'embrasement général. La parole baroque constitue un de ces moyens mais elle peut conduire à la violence. Certes cet échange est un moyen de reconnaître l'autre comme partenaire digne de soi en même temps qu'on cherche à le provoquer, à le défier, à l'humilier ou à la limite à le tuer. Mais l'absence d'une autorité extérieure reconnue, hors jeu en quelque sorte, pour réguler l'affrontement entre les proches, ou entre les factions politiques au niveau du pays, expliquerait très vraisemblablement les dérapages 
extrêmes qu'a connu le Liban. Par ailleurs, il n'existe pas, comme dans les pays démocratiques, une idéologie de la souveraineté monarchique ou républicaine pour coiffer les luttes politiques, contrôler le jeu du pouvoir et I'orienter vers d'autres finalités. Au Liban, le politique est une forme de lutte sans alternative 13. C'est cela qui fait à la fois son originalité et son malheur.

\section{Bibliographie}

Campbell J. K., 1964. Honor, Family and Patronage. A Study of Institutions and Moral Values in a Greek Mountains Community, Oxford, Clarendon Press.

Eddé D., 1989. Lettre posthume, Paris, Gallimard.

Gilsenan M., 1996. Lords of the Lebanese Marches. Violence and Narrative in an Arab Society, London, IB Taurus Publishers.

Jamous R., 1981. Honneur et baraka. Les structures sociales traditionnelles dans le Rif, Paris, Cambridge University Press / Maison des sciences de I'homme.

1993. «Mensonge, violence et silence dans le monde méditerranéen », Terrain, $\mathrm{n}^{\circ} 21$, pp. 97-110.

Johnson M., 1977. «Political bosses and their gangs: Zu'ama and qabadayat in the Sunni Muslim quarters of Beirut», in Gellner E. \& J. Waterbury (ed.) Patrons and Clients, London, Duckworth, pp. 207-224

Khalaf S., 1977. "Changing forms of political patronage in Lebanon », in Gellner E. \& J. Waterbury (ed.) Patrons and Clients, London, Duckworth, pp. 185-206.

\section{Notes}

1 Cf. I'article de M. Johnson (1977) sur la position et le rôle des zu'ama (singulier : za'im) urbains équivalents des beys d'origine rurale et des qabaday, les « hommes forts », leurs hommes de main, et celui de S. Khalaf (1977) qui montre comment les grandes familles maronites, druzes ou sunnites ont continué à exercer leur pouvoir dans la montagne libanaise et comment, après la fin de la période ottomane, elles se sont adaptées à l'occupation française et à l'indépendance du pays.

2 Généralement Gilsenan présente chaque narration par un synopsis, puis donne en détail son déroulement et les commentaires qui $s^{\prime} y$ rapportent. Il y ajoute ses analyses qui sont une sorte d'explication de texte permettant au lecteur de 
comprendre ce qui est dit et parfois même ce qui ne se dit pas ou ne doit pas être dit. Ces interprétations introduisent à d'autres parties du récit et à d'autres commentaires. Les commentaires de Gilsenan constituent une analyse fine des conflits, de la vengeance, de la manière dont I'univers des relations sociales évolue, dont s'élaborent les stratégies, etc.

3 Chez les gens du Akkar, les récits narratifs ne sont pas simplement une manière d'interpréter les faits sociaux, ils sont, comme on l'a vu plus haut, une forme d'action et d'interaction, une forme d'échange social. L'anthropologue s'adresse à un lecteur absent à qui il livre un écrit, une analyse de ce qu'il a vu et entendu, à qui il essaie de communiquer ce qu'il a compris. Appeler «narration » ce que font les hommes locaux et ce que dit l'anthropologue, n'est-ce pas confondre sous un même vocable des choses hétérogènes ?

4 Ce travail fait suite à un premier essai comparatif sur les rapports entre violence physique et usages de la parole dans le monde méditerranéen (Jamous 1993 : 97110).

5 Gilsenan a choisi de commencer par l'analyse du pouvoir et de son évolution avant de considérer les autres aspects des relations de violence, ce qui, de mon point de vue, affaiblit son propos, d'autant que les données sur les autres acteurs sont dispersées dans l'ouvrage.

6 Le terme de shab (pluriel : shebab) que I'on traduit par « jeune » ne correspond pas à une catégorie d'âge. Il existe dans cette région du monde des hommes mariés, pères de famille, âgés de 50 ans et que I'on appelle «jeunes ». Le terme s'applique à un statut social comme on le verra dans cette partie.

7 On peut faire I'hypothèse que les paysans ont moins le sens de I'honneur que les aghas et acceptent de recevoir une compensation plutôt que de se venger.

8 Une comparaison avec l'exemple de nomades sarakatsani étudiés par J. Campbell est éclairante. En effet, dans cette communauté grecque, un vieux ne participe plus au jeu de l'honneur, laissant à ses enfants adultes le soin de le faire. Il se préoccupe de préparer sa mort et son salut éternel (dans le monde de l'au-delà). Il est déjà hors-du-monde. On ne sait pas si, au Liban, des vieux font de même. Il semble qu'ils se mettent au service du lignage.

9 Dans l'autre récit (voir note 5), c'est le compagnon, parent lointain du jeune homme, qui, en désignant le meurtrier de son oncle paternel, apparaît comme le manipulateur d'une personne qui réagit vite sans réfléchir et qui, après sa sortie de prison, aura un comportement jugé bizarre.

10 Cette citation concerne un autre jeune que M. Gilsenan rapproche de celui dont nous parlons, et qui va provoquer un bey. Son action sera catastrophique puisque son frère (qui cherchait à le calmer) sera tué dans une confrontation violente avec ce bey et lui-même sera sérieusement blessé. Il est significatif qu'aucune action ne fut entreprise pour le venger par ses agnats de son segment de lignage et encore moins par ceux de l'autre segment (Gilsenan 1996 : 258-261).

11 Certes tous les beys ne vont pas jusqu'à cet extrême, mais il n'est pas rare que la mémoire locale retienne comment certains beys marquent leur action de manière transgressive. Ne raconte-t-on pas qu'un vieux bey se fit sodomiser par un de ses serviteurs «parce que c'était son bon plaisir» et non par comportement homosexuel ? (190).

12 Certes la situation chez les sunnites du Akkar n'est pas celle des maronites, des druzes ou des chiites qui disposent à des degrés divers d'un clergé ou d'une autorité 
religieuse affirmée. Il faudrait de nombreuses études pour savoir si ces hommes de religion interviennent de manière différente de ceux du Akkar. Notre hypothèse est qu'il y a des différences de degrés dans la relation entre religion et politique selon les communautés et non une différence fondamentale.

13 Il me paraît faux de penser que la guerre civile libanaise se ramène à un affrontement entre communautés religieuses. C'est souvent à l'intérieur de ces communautés que la violence fut la plus terrible et l'ouvrage de Gilsenan est là pour nous en montrer les prémices. 